\title{
Atypical cystic parathyroid adenoma masquerading as a thyroid nodule and presenting with severe hypercalcemia
}

\begin{abstract}
Background: Primary hyperparathyroidism occurs as a result of isolated parathyroid adenoma in $80 \%$ to $85 \%$ of all cases. Severe hypercalcemia due to cystic atypical parathyroid adenoma is rare entity.

Method: We report a rare case of severe hypercalcemia associated with cystic atypical parathyroid adenoma.

Result: 27 year old female presented with nausea, vomiting and abdominal pain. A serum calcium level of $15.8 \mathrm{mg} / \mathrm{dl}$ with PTH of 413 was noted on admission. She was initially treated with IV hydration and had ultrasound of neck which showed $2.8 \mathrm{~cm}$ complex nodule in right thyroid lobe. After appropriate work up, she underwent a surgical resection of parathyroid adenoma found on CT with 3D reconstruction. Pathology showed a mixed picture more consistent with possible atypical adenoma.
\end{abstract}

Conclusion: Parathyroid cyst is known but rare entity, which is usually nonfunctional. However, atypical cystic parathyroid adenoma with severe hypercalcemia is very rarely reported. It may exhibit some pathologic features of carcinoma, but angio-invasion and/or metastases are not present. Severe hypercalcemia with a large, cystic neck mass should prompt thoughts of cystic atypical parathyroid adenoma versus carcinoma. In both cases, long-term follow up would be prudent. Also, our case emphasizes the importance of knowing ultrasonographic features to differentiate parathyroid adenoma appearing as thyroid nodule.

Keywords: atypical adenoma; hypercalcemia; parathyroid adenoma; thyroid nodule; ultrasonographic features; hypercalcemia; cystic neck mass; ultrasound; thyroglobulin; parathyriodectomy; hyperparathyroidism; neoplasm; vitamin $\mathrm{d}$; immunohistochemistry; carcinoma
Volume 2 Issue 4 - 2015

\section{Manisha Garg, Nitesh D Kuhadiya, Sartaj Sandhu,Aditya Mehta Manav Batra, Ajay Chaudhuri}

Department of Endocrinology-Diabetes and Metabolism, University at Buffalo, USA

Correspondence: Nitesh D Kuhadiya, Department of Endocrinology-Diabetes and Metabolism, University at Buffalo, 462 Grider Street, ECMC, 3rd Floor, Ambulatory Care Center, NY 142I5, USA, Tel 630056856,Email niteshku@buffalo.edu

Received: August 29, 2015 | Published: November 02, 2015
Abbreviations: HPT, hyperparathyroidism; PHPT, primary hyperparathyroidism; IV, intravenous

\section{Introduction}

Primary hyperparathyroidism (PHPT) is the most common cause of hypercalcemia. Majority of cases are related to single gland adenoma. Parathyroid carcinoma, atypical parathyroid adenoma, parathyroid cysts and parathyromatosis are rare conditions that account for approximately $2 \%$ of all patients with PHPT. We report an unusual case of severe hypercalcemia due to cystic atypical parathyroid adenoma, ${ }^{1}$ which appeared as thyroid nodule on ultrasound. Such a case is rarely described in literature.

\section{Case report}

A 27-year old Caucasian woman with history of mental retardation presented with severe nausea, vomiting and abdominal pain of 1 day duration. No similar episodes were reported in the past. There was no prior history of renal stones or fractures. On examination, she was afebrile, with BP 130/75mm Hg and HR 90-100 beats/minute. She was confused and had generalized tenderness over her abdomen. Laboratory data (Table 1) revealed hypercalcemia secondary to primary hyperparathyroidism. Neck ultrasound (Figure 1A \& 1B) revealed a right dominant $2.8 \mathrm{~cm}$ complex, solid and cystic nodule with intranodular and peripheral vascularity. She was admitted and was treated with intravenous (IV) normal saline. She received 1 dose of IV pamidronate $60 \mathrm{mg}$. Her calcium level improved to $9.8 \mathrm{mg} / \mathrm{dl}$ and was subsequently discharged. She underwent biopsy of the right thyroid nodule, which was consistent with parathyroid neoplasm possibly adenoma with immunohistochemistry negative for Thyroglobulin, TTF 1, Calcitonin and S 100. She was subsequently referred to endocrine surgery for evaluation of parathyriodectomy. CT scan of neck with 3D imaging reconstruction ${ }^{2}$ (Figure 2) study confirmed a large well defined nodule with peripheral enhancement suspicious for large right parathyroid adenoma. She underwent minimally invasive parathyriodectomy with fluid aspiration and intraoperative PTH levels fell from $289 \mathrm{pg} / \mathrm{ml}$ to $15.8 \mathrm{pg} / \mathrm{ml}$. Pathology (Figure 3) was supportive of the diagnosis of atypical parathyroid adenoma with fragments of hyper cellular parathyroid neoplasm consisting of neoplastic cells with solid nest and trabecular growth patterns with focal capsular distortion and increased mitotic activity. Immunohistochemistry revealed Ki-67 proliferation index 1-3\%, bcl-2 positive, diffuse strong and focal weak positive $\mathrm{p} 53$. Post operatively; she was started on calcium and vitamin D supplementation. She is doing well and remains normocalcemic for 2 years following surgical resection with most recent calcium of $9.3 \mathrm{mg} / \mathrm{dl}$. 

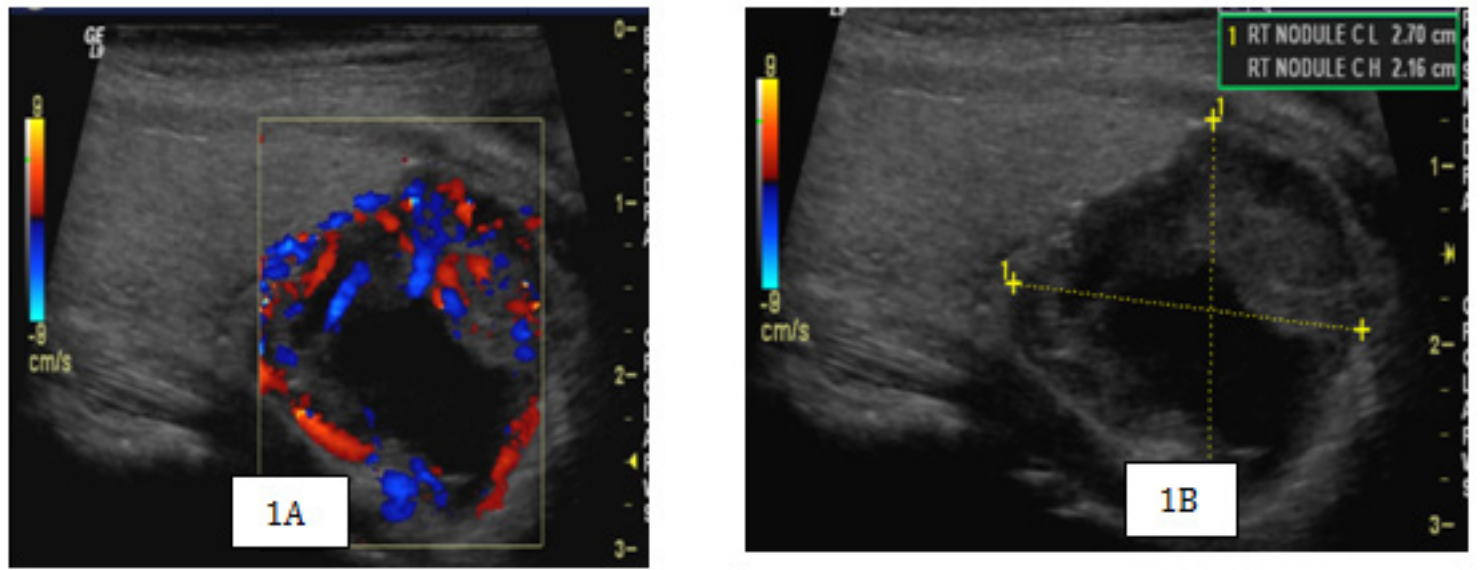

Figure I A:Ultrasonographic findings consistent with right thyroid nodule with solid and cystic component. IB:prominent peripheral and intranodular vascularity.
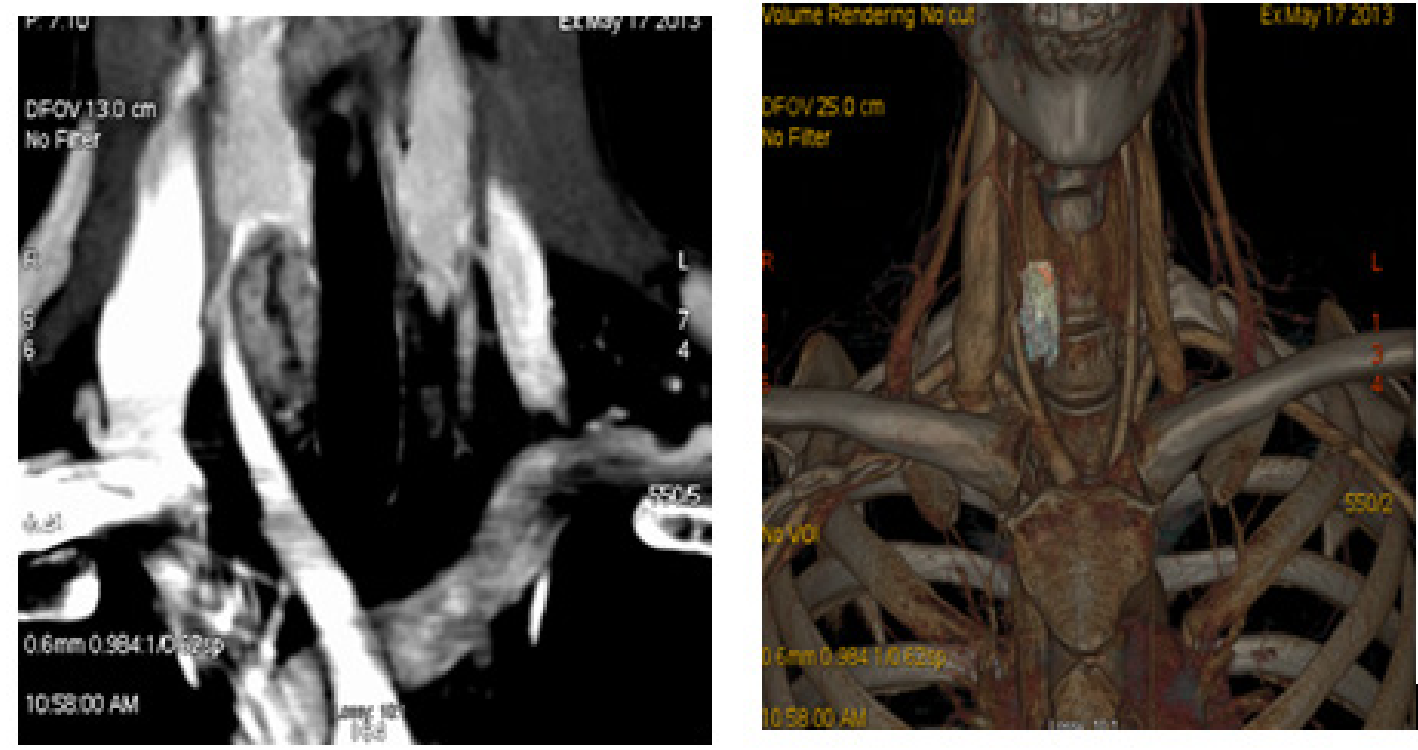

Figure 2 Localization of parathyroid adenoma on CT of neck with 3 D reconstruction.
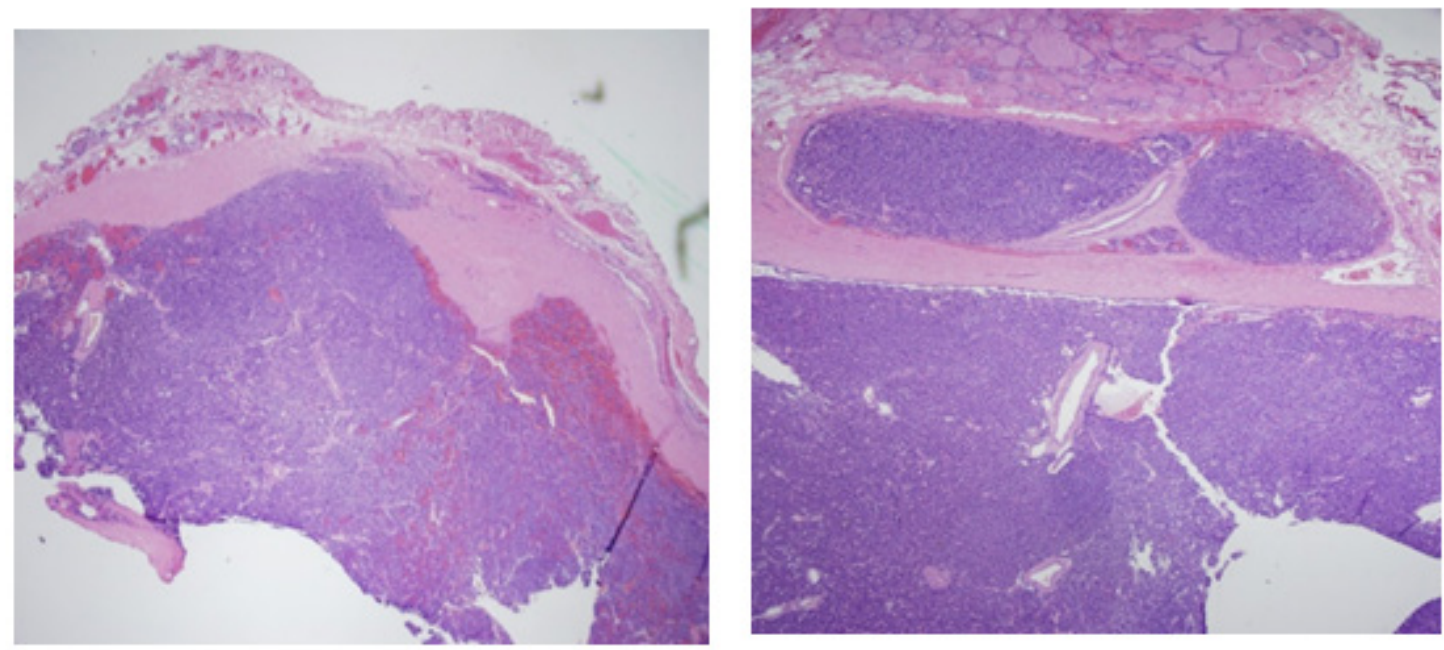

Figure 3 Hyper cellular neoplastic cells with solid nest and trabecular growth pattern separated by thick fibrous tissue band.

Citation: Garg M, Kuhadiya ND, Sandhu, et al.Atypical cystic parathyroid adenoma masquerading as a thyroid nodule and presenting with severe hypercalcemia. Endocrinol Metab Int J. 20I5;2(4):I3 I-I34. DOI: I0.I5406/emij.20I5.02.00028 
Table I Laboratory parameters

\begin{tabular}{lll}
\hline Labs & Admission & Post-surgery \\
\hline Total Ca & $15.8(8.5-10.5 \mathrm{mg} / \mathrm{dl})$ & $9.4 \mathrm{mg} / \mathrm{dl}$ \\
lonized Ca & N/A & \\
PTH Intact & $413(12-72 \mathrm{pg} / \mathrm{ml})$ & $\mathrm{I}$ \\
$25-\mathrm{OH}$ Vitamin D & $9(30-100 \mathrm{ng} / \mathrm{ml})$ & $23 \mathrm{ng} / \mathrm{ml}$ \\
I-25 HydroxyVitamin D & N/A & $3.8 \mathrm{mg} / \mathrm{dl}$ \\
Phosphorous & $3(2.5-4.8 \mathrm{mg} / \mathrm{dl})$ & \\
24 Hour Urinary Calcium & N/A & \\
BUN/Creatinine/E-GFR & $16 / 0.76 />60$ & $138 \mathrm{mmol} / \mathrm{L}$ \\
Sodium & I $44(135-145 \mathrm{mmol} / \mathrm{L})$ & \\
\hline
\end{tabular}

\section{Discussion}

Parathyroid cysts are rare lesions found in neck and anterior mediastinum. They are often nonfunctional $(>90 \%)$ and rarely in functional form. ${ }^{3-6}$ However, cases of sever hypercalcemia with calcium level of $15-23 \mathrm{mg} / \mathrm{dl}$ due to parathyroid cysts have been reported. . $3-5,7,8$ Formation of parathyroid cysts remain a question and different hypothesis have been postulated including persistence of vestigial branchial clefts/Kurtsteiner canal. Many investigators believe that functional cysts are due to degenerative changes in preexisting parathyroid adenoma. ${ }^{9-11}$

Similarly, atypical adenoma is a rare cause of primary hyperparathyroidism. It is certainly uncommon for an atypical parathyroid adenoma to present with severe hypercalcemia, although calcium level of 23.3 has been reported by due to an adenoma. ${ }^{12}$ The majority of patients with parathyroid carcinoma and atypical adenoma can be easily distinguished from patients with primary hyperparathyroidism due to classic adenoma, but distinguishing these conditions from each other is more difficult because of lack of precise clinical and histologic criteria. Those tumors with worrisome features but not diagnostic of malignancy, fall under category of "atypical adenoma." Clinically, parathyroid cancer more commonly presents with palpable neck mass, hoarseness of voice and profound Hypercalcemia. $^{13}$ Histopathologically, atypical adenoma and parathyroid cancer share features such as fibrous capsule, fibrous trabeculae, trabecular growth pattern and mitotic figures, however true capsular, tissue and vascular invasion along with recurrence is exclusive to parathyroid cancer. ${ }^{14}$ Existence of local recurrence or metastatic disorder is the single and reliable characteristic in distinguishing between benign and malignant parathyroid disorders. ${ }^{15}$

Our patient had thick capsule with some focal distortion associated with mitotic activity but no vascular or local invasion was noted. Although, our patient had positive markers, the role of prognostic markers as $\mathrm{Ki}-67$, p53, bcl-2 that may assist in evaluating aggressive behavior in these tumors has not been fully studied and is limited because of lack of consistent difference in their expression. ${ }^{16}$ Absence of nuclear staining for parafibromin and presence of protein gene product 9.5 (PGP 9.5) has been postulated as diagnostic of parathyroid carcinoma. Also, some patients with sporadic parathyroid carcinoma have germline HRPT2 mutations, ${ }^{17}$ but unfortunately, in our case, both parafibromin/PGP 9.5 staining and genetic testing were not done. Interestingly, our patient had both rare features of 'cyst' and 'atypia' together. Co-existence of these two entities makes this case extremely rare. To our knowledge, so far only one similar case has been described in literature. It is important to report such cases to estimate the true prevalence of cystic atypical parathyroid adenoma and broaden the differential diagnosis of a cystic mass with hypercalcemia.

Also, in our patient, the parathyroid adenoma was masquerading as a thyroid nodule on ultrasound images. Certain ultrasound characteristics used to distinguish a parathyroid adenoma from thyroid nodule include solid composition, hypo echoic appearance and presence of a feeding polar vessel. ${ }^{18}$ Ultrasound is inexpensive and noninvasive technique for localization but it needs experienced hands to identify and distinguish parathyroid adenoma from thyroid pathology. Sometimes, US directed biopsy with PTH analysis is required for confirmation.

\section{Conclusion}

Atypical cystic adenoma are rare, they have some features of carcinoma but lacks the clear evidence of malignancy like invasion or metastasis. They have unpredictable clinical course, hence longterm follow up is prudent to assess for local recurrence and distance metastases to distinguish between benign and malignant parathyroid disease. Also, it can rarely present with severe hypercalcemia as in our case. This case also highlights the importance of suspecting parathyroid adenoma mimicking a thyroid nodule and emphasizes on appropriate follow up imaging or procedure for confirmation.

\section{Acknowledgments}

None.

\section{Conflicts of interest}

The auhtor declares there is no conflicts of interest.

\section{References}

1. Calandra DB, Shah KH, Prinz RA, et al. Parathyroid cysts: a report of eleven cases including two associated 5 with hyperparathyroid crisis. Surgery. 1983;94(6):887-892.

2. Wani S, Hao Z. Atypical cystic adenoma of the parathyroid gland: case report and review of literature. Endocr Pract. 2005;11(6):389-393.

3. Ginsberg J, Young JE, Walfish PG. Parathyroid cysts. Medical diagnosis and management. JAMA. 1978;240(14):1506-1507. 
4. Ihm PS, Dray T, Sofferman RA, et al. Parathyroid cysts: diagnosis and management. Laryngoscope. 2011;111(9):1576-1578.

5. Stoffer SS, Hawker CD. Parathyroid cysts--medical diagnosis and management. JAMA. 1979;241(4):357.

6. Wu W. The diagnosis and surgical management of parathyroid cysts. Zhonghua Wai Ke Za Zhi. 1995;33(11):673-674.

7. Carroll MF, Schade DS. A practical approach to hypercalcemia. Am Fam Physician. 2003;67(9):1959-1966.

8. Van den Hauwe K, Oeyen SG, Schrijvers BF, et al. A 50-year-old man with severe hypercalcemia: a case report. Acta Clin Belg. 2009;64(5):442-446.

9. Glynn N, Cunningham J, Tormey W, et al. Multiple functional parathyroid cysts. J Clin Endocrinol Metab. 2013;98(7):2641-2642.

10. McCoy KL, Yim JH, Zuckerbraun BS, et al. Cystic parathyroid lesions: functional and nonfunctional parathyroid cysts. Arch Surg. 2009;144(1):52-56.

11. Margolis IB, Wayne R, Organ CH. Parathyroid cysts: Functional and mediastinal. Surgery. 1975;77(3):462-466.

12. Mishra A, Newman D. An Interesting Case of Life-Threatening Hypercalcemia Secondary to Atypical Parathyroid Adenoma versus Parathyroid Carcinoma. Case Rep Med. 2014;2014:473814.
13. Fernandez-Ranvier GG, Khanafshar E, Jensen K, et al. Parathyroid carcinoma, atypical parathyroid adenoma, or parathyromatosis? Cancer 2007; $110: 255-264$.

14. Ippolito G, Palazzo FF, Sebag F, et al. Intraoperative diagnosis and treatment of parathyroid cancer and atypical parathyroid adenoma. $\mathrm{Br}$ J Surg. 2007;94(5):566-570.

15. Flye MW, Brennan MF. Surgical resection of metastatic parathyroid carcinoma. Ann Surg. 1981;193(4):425-435.

16. Vargas MP, Vargas HI, Kleiner DE, et al. The role of prognostic markers (MiB-1, RB, and bcl-2) in the diagnosis of parathyroid tumors. Mod Pathol. 1997;10(1):12-17.

17. Howell VM, Gill A, Clarkson A, et al. Accuracy of combined protein gene product 9.5 and parafibromin markers for immunohistochemical diagnosis of parathyroid carcinoma. J Clin Endocrinol Metab. 2009;94(2):434-c441.

18. Heller MT, Yip L, Tublin ME. Sonography of intrathyroid parathyroid adenomas: are there distinctive features that allow for preoperative identification? Eur J Radiol. 2013;82(1):e22-e27. 